\title{
The Disaster of Costa Concordia Cruise Ship: an Accurate Reconstruction Based on Black Box and Automation System Data
}

\author{
Paolo Gubian, Mario Piccinelli \\ Dept. of Information Engineering \\ University of Brescia \\ Brescia, Italy \\ paolo.gubian@unibs.it \\ mario.piccinelli@gmail.com
}

\author{
Paolo Neri \\ Dept. of Civil and Industrial Engineering \\ University of Pisa \\ Pisa, Italy \\ paolo.neri@for.unipi.it
}

\author{
Bruno Neri \\ Dept. di Ingegneria dell'Informazione \\ University of Pisa \\ Pisa, Italy \\ b.neri@iet.unipi.it
}

\author{
Francesco Giurlanda \\ Consorzio di Ricerca per le Telecomunicazioni \\ Pisa, Italy \\ francesco.giurlanda@coritel.it
}

\begin{abstract}
In this paper, an accurate reconstruction of the events preceding the January $13^{\text {th }}, 2012$ impact of the Costa Concordia cruise ship with the rocks of Isola del Giglio is presented, along with the emergency countermeasures activated by the ship automation system after the impact. The reconstruction is entirely based on data recorded by the information systems of the ship and demonstrates the importance of this kind of data from a scientific and forensics point of view. First the authors, three of whom have served as consultants in the trial in Grosseto, Italy, show how information stored in the Voyage Data Recorder, the so called "Black Box", has been used to calculate the exact time and coordinates of the impact point. An accurate evaluation of these data represents a "conditio sine qua non" to ascertain the effects of the helmsman's error, who allegedly misunderstood the Master's orders just 20 seconds before the impact. Then, these effects were studied by means of a purposely developed short term manoeuvring simulator, whose results are also briefly summarized in the paper. Moreover, information stored in the hard disk of the automation system of the ship, concerning the activation of emergency source of energy, have been examined and some results are briefly reported in the paper. The most important data are those concerning the watertight doors, the passenger elevators and the Emergency Diesel Generator. According to the results of our investigations, i) the helmsman's error appears to have been determinant in the accident; ii) the emergency procedure, which started automatically after blackout of the main power source, does not appear to have performed correctly. In our opinion, both these facts could have influenced the outcome of the last phase of the ship evacuation, during which most of the victims lost their lives.
\end{abstract}

Keywords- Costa Concordia; shipwreck; manoeuvres simulator; VDR; black box.

\section{INTRODUCTION}

A detailed reconstruction of the events preceding and following a great disaster like that of the Costa Concordia shipwreck is a very complex task. The accident occurred on January $13^{\text {th }}$, 2012, just in front of the coast of Isola del Giglio (Tuscany). Three independent inquiries were conducted, by the Italian Coast Guard, by the Italian Justice, and by the IMO (International Maritime Organization). A complete analysis of the resulting data is essential not only for forensic purposes, but also to ascertain all the causes of the accident and possibly also to suggest improvements to emergency equipment and procedures. In the days immediately following the shipwreck, rather simplistic reconstructions of the event appeared in the media, in which the whole responsibility was being attributed to the ship's master, Francesco Schettino. Additional elements regarding the behaviour of other crew members and persons involved, as well as some apparent malfunctions of the emergency systems aboard, were not known or not taken into account, thus giving an incomplete picture of the accident. During the trial in Grosseto, an extraction of the data stored inside the hard disks of the information systems of the ship was carried out. The information extracted concerned not only the events occurred just before the impact, but also what happened up to the abandon of the ship. The results were rather surprising and additional facts emerged. For instance, 
from a comparison between the audio files and the instantaneous position of the rudders of the ship, both recorded in the black box, it was possible to determine that the helmsman did not correctly execute the captain's orders only 20s before the impact. After this, a specially developed manoeuvre simulator stated that this error has had a significant effect in the accident. Moreover, data stored in the hard disks of the automation system of the ship confirmed that, as reported in many testimonies, the emergency generator did not operate correctly after the blackout, caused by the flooding of the main power sources of the ship. From these data, it was also possible to extract useful information about the mandatory tests which were periodically performed on this equipment.

The paper illustrates the reconstruction of the events based on the data stored in the information systems of the ship. It is organized as follows. In Section II, a brief description of the two main sources of data used for the aforementioned analysis is given. Then, the method with which these data have been used for an accurate reconstruction of the impact is described in Section III. Section IV contains a brief presentation of the short term manoeuvre simulator built during the trial by the authors, in order to evaluate the effects of the helmsman's error. Section V presents the reconstruction of some events concerning the automatic emergency procedure, namely those regarding the emergency generator; finally some conclusions are drawn in Section VI.

\section{VOYAGE DATA RECORDER AND AUTOMATION SYSTEM}

International regulations about the safety of ships at sea require every modern ship to be equipped with a Voyage Data Recorder (VDR) to assist investigations in the event of an accident. Voyage Data Recorders are systems installed on modern vessels to record data related to the ship's status and thus provide data to investigators in the case of an accident. While the data collection is performed by different devices, such as analog and digital sensors or dedicated computer systems, the data recording is usually performed by an industrial grade computer.

According to the regulations, a standard VDR should store at least the following data items: date and time (referenced to Coordinated Universal Time UTC), position (latitude, longitude, coordinate reference), speed, heading, bridge audio (through one or more microphones that record conversations and audible alarms), VHF (Very High Frequency band) radio communications, the preferred way for maritime communications, radar data (faithful replicas of radar display at the time of recording), depth under keel, main alarms, rudder orders and responses, engine orders and responses, hull openings status, watertight and fire door status and, where available, accelerations and hull stresses.

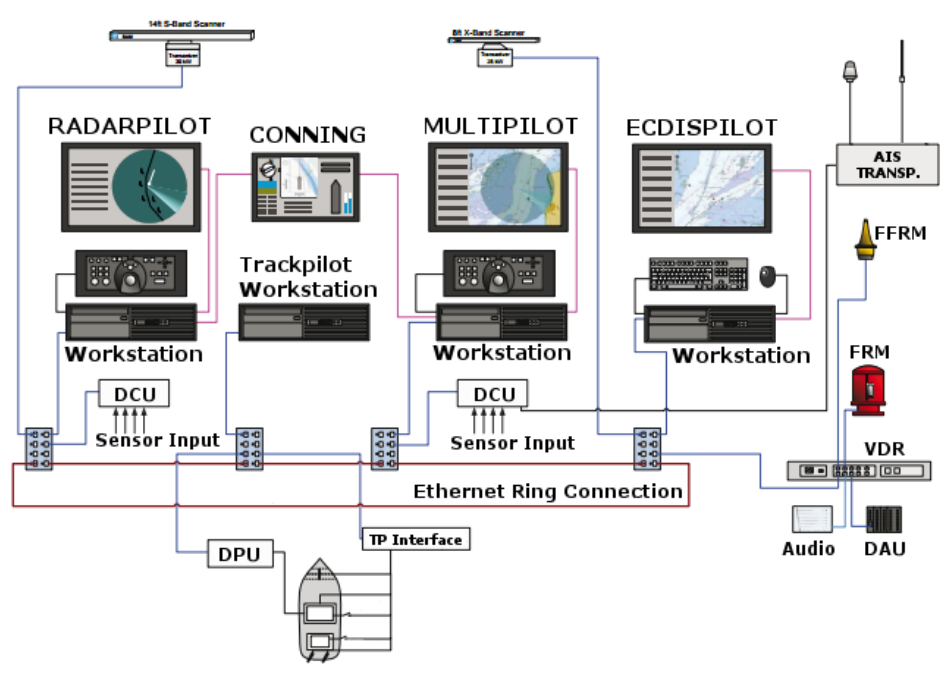

Fig. 1 Example of an Integrated Navigation system similar to the one installed on the Costa Concordia bridge

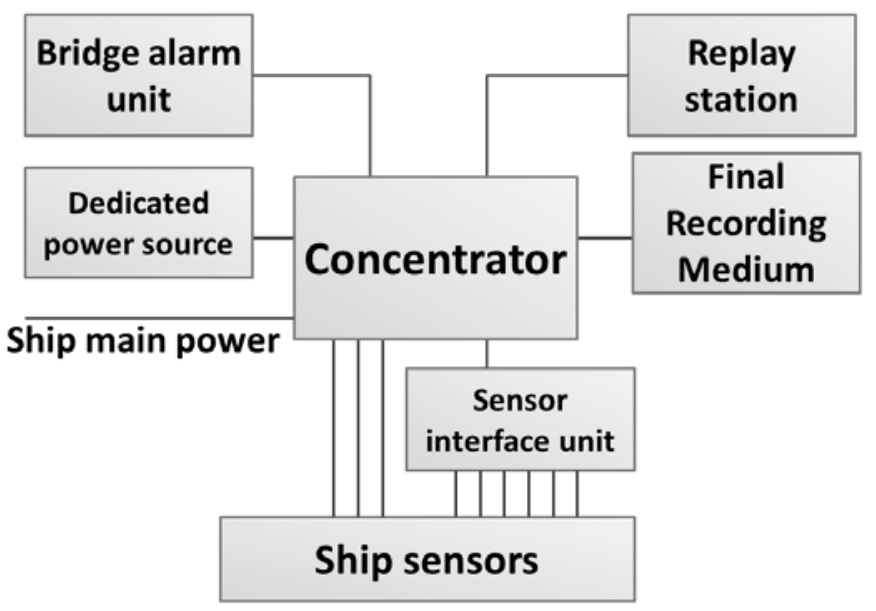

Fig. 2 Voyage Data Recorder (VDR) system architecture

The VDR is the complete system for processing, encoding and recording the data required by the IMO (International Maritime Organization) regulations. On modern ships, the VDR is integrated into a complete integrated navigation system, as shown in Figure 1. The elements of a VDR, as seen in Figure 2, are:

- $\quad$ Concentrator: usually an industrial grade computer which acquires data from sensors, processes and encodes them and records the stream to the final recording medium.

- Sensors: external devices from which the VDR acquires data.

- $\quad$ Sensor interface unit: optional device providing additional input lines to the concentrator.

- $\quad$ Final recording medium (FRM): the capsule used to store the data, designed to survive an accident and thus enable the recovery of the voyage data even in the event of a catastrophic loss of the ship. This is the part which is often referred to as the "Black Box". 
- Dedicated power source: an external battery exclusively used to power the VDR for at least 2 hours in case the ship loses both main and backup power.

- $\quad$ Bridge alarm unit: a remote interface to manage the VDR and acknowledge system alarms and warnings.

- Replay stations: one or more optional personal computers used to download and review voyage data from the concentrator.

In the case of an accident and subsequent investigation, both the final recording medium and, if they survived, the concentrator and the replay stations can serve as sources of data. The final recording medium must store at least the last 12 hours of data. Recording must continue until 2 hours after the loss of ship power.

The VDR main unit is wired to all the devices which provide data. Data sources can be split into categories, depending on the interface:

- Microphones: analog signals from bridge microphones, bridge telephones and VHFs: usually sampled by the concentrator.

- Digital signals: state signals carrying information about the ship's alarms and warnings.

- Analog signals: signals acquired from analog transducers: for example, information about position of the rudders.

- Video signals: signals from analog video cameras, usually to record the radar display for ships equipped with older analog radar devices.

- $\quad$ Serial data: data generated by smart devices such as the weather station, digital radar, AIS () etc. Communication usually consists of NMEA strings (discussed shortly). Sensor interface units usually convert their analog/digital/serial input data into NMEA strings, which are then fed to the main unit.

- $\quad$ Complex data: bidirectional data connections (usually Ethernet) to autonomous devices providing complex data for the VDR to store. As an example, modern ships feature radar stations which are themselves industrial computers and concentrate all the information available on the bridge; these stations are able to provide the VDR with, for example, digital screenshots of radar displays.

On modern ships all of these feeds are stored in three formats: images (screenshots from the radar display(s), audio files (from microphone inputs) and text (NMEA strings representing serial, analog and digital inputs).

The preferred way to store voyage data into the VDR is via NMEA strings. NMEA 0183 [1] is a combined electrical and data specification for communication among marine electronic devices such as echo sounder, sonar, anemometer, gyrocompass, autopilot, GPS receivers and many others. It has been defined by, and is controlled by, the U.S.-based National Marine Electronics Association.

An example of an NMEA string carrying information about the location of a generic GPS receiver is:

\$GPGGA,123519,4807.038,N,01131.000,E,1,08,0.9,545.4,M, 46.9,M, $* 47$

The elements composing the string are the following:

- $\quad$ \$: start symbol.
- GP: first two characters of preamble identify the data source (GP for GPS receiver).

- GGA: last three characters of the preamble give a standard description of the following data (GGA for global positioning fix data).

- 123519: time when the fix was taken (12:35:19

UTC).

- $\quad$ 4807.038,N: latitude (48 deg. 07.038’ North).

- $\quad$ 01131.000,E: longitude (11 deg. 31.00’ East).

- $\quad$ 1: fix quality (1 for GPS fix).

- $\quad$ 08: number of satellites being tracked.

- 0.9: horizontal dilution of precision (index of the precision of the satellite positioning)

- 545.4,M: height above mean sea level (545.4 meters).

- 46.9,M: height of mean sea level above WGS84 ellipsoid.

- $\quad$ Empty field: time in seconds since last update.

- $\quad$ Empty field: DGPS station ID number.

- $\quad$ *47: checksum data (always begins with *).

One of the biggest problems in VDR forensics is trying to find the meaning of the data contained in non-standard NMEA sequences, which the standard allows equipment manufacturers to define. This goal can be accomplished either with the help of the manufacturer, or if this is not available for any reason, by deduction and by combining data from other similar sources.

Investigation of the accident focused mainly on evidence extracted from the Voyage Data Recorder. While the FRM unit was found to be not working, luckily the concentrator survived the accident and enabled investigators to retrieve all the required data.

The disk inside the concentrator was recovered and acquired by Italian police and the disk image was made available to all the involved parties. The disk content was not immediately useful as it was structured in a way not easy to be read without a proprietary software. The first step in the investigation was then to discover the format of the stored elements and then to build an ad-hoc suite of software tools to automate the recovery process.

The disk is an 80 GB device with a single non-bootable partition formatted in the QNX 4.0 file system.

Our analysis began from the "frame" and "NMEA" directories, which as we presumed, and later confirmed, contained the files related to the radar screenshots and to the NMEA sequences archive.

In these directories we found a large number of files, each one without extension and named in numerical order (0000001, 0000002, etc.), which did not bear any recognizable header. Further analysis with an hex editor revealed that the files were in fact GZip compressed files preceded by a nonstandard header containing what was supposed to be the real name of the file, with extension .BMP.GZ for radar frames and .LOG.GZ for NMEA files.

Starting from this knowledge we wrote Bourne Shell and Python scripts to extract and decompress the embedded files and rename them with their real name. At the end of the recovery we were able to obtain: 
- $\quad$ Radar screenshots, stored as bitmap images. These had been collected from the two main radar displays of the bridge, alternating in intervals of about seven seconds. The real names of the files contained an indication to which radar display the image was taken from. In total, the folder contained 11,759 images spanning for about 24 hours preceding the VDR shutdown, hence from January 12th at 23:06 to January 13th at 23:36 (local time, UTC+1).

- $\quad$ NMEA strings, stored as ASCII text files. Each text file contains a number of long rows. Each row consists of a timestamp, in UNIX epoch format (number of seconds since midnight, January 1st, 1970) in hexadecimal format, followed by some NMEA strings which had supposedly been collected at that time. Each file holds strings related to a 5 minutes time span, ranging for about a week preceding the VDR shutdown, hence from January 6th at 22:50 to January 13th at 23:35 (in local time, UTC+1). As in the case of radar images, the file namespace is used as a circular buffer [2].

After retrieving the data in a useful format, the subsequent steps were directed towards finding ways to concentrate these data to show specific aspects of the accident, deemed useful for the investigation. These aspects, which will be presented in the following sections, are the rudder status, in the minutes before and after the impact, the status of watertight doors during navigation and a check of the route that had been set on the autopilot upon departure.

The Martec Security Management System (SMS) is the safety supervisor, which collects and manages the safety information coming from all the onboard safety systems: fire detection system, emergency shutdown system, watertight door system and hi-fog system. The SMS forwards a subset of the safety information it manages to the VDR, in order to have it recorded. The information is sent using the NMEA-0183 protocol (equivalent to IEC 61162-1). Since the NMEA standard does not define specific sentences describing safety information, Martec SMS defined some proprietary sentences, and provided the documentation needed to decode and understand the proprietary NMEA sentences stored into the VDR. Unfortunately, this information allowed only a partial reconstruction of the events. Martec SMS stores the complete set of safety information into proprietary log files. These files are stored in a hard disk as a set of 21 binary files named according to a scheme like YYYYMMDD.SBL, and cover a period from $12 / 25 / 2011$ to $1 / 14 / 2012$. Access to this information required the active collaboration of Martec, which used a proprietary software to extract the data from the log files. Martec provided only the data from 1/12/2012 to $1 / 14 / 2012$, since the other information was deemed out the scope of the investigation. But the failure of the Emergency Diesel Generator (EDG), documented in the VDR data and in testimonies, required a more in-depth investigation of the tests which were periodically performed on EDG. If the tests were performed correctly, they would appear in the SMS logs. Thus, we tried to extract the previous log files ourselves. We were able to decode the binary log files by comparison with the data extracted by Martec, using a technique similar to what is known as a KPA (Known-plaintext Attack) cryptanalysis literature [3]. The analysis showed that each log file presented a header of 4 bytes followed by a sequence of events. The file header is always the same and is equal to 0x0152424C, the sequence of bytes identify a SBL file. Each event is formatted as follow:

\section{$<$ EH, Sensor ID, 0x00, State, 0x00, Description, 0x00 >}

Where $\mathrm{EH}$ is the event header which consists of a constant sequence of 21 bytes. It keeps the time information of the event, encoded as a UNIX timestamp (integer number of seconds elapsed since midnight, Jan 1, 1970 UTC). Sensor ID, State and Description are respectively the identifier of the sensor that triggered the event, the state of the sensor and a brief description of the event. Due to dynamic length of these fields, a separator byte (0x00) was used to identify when each field ends.

The analysis allowed us to retrieve all the information about the EDG from the 25/12/2012, specifically: when the test were performed, how many minutes they lasted, all the events concerning the EDG room, such as ignition and shutoff of the fans.

\section{IMPACT RECONSTRUCTION}

During the trial in Grosseto, it emerged that the helmsman made a significant error some 20 s before the impact, misunderstanding the order of captain Schettino and putting the rudder "hard to starboard" instead of "hard to port". This was demonstrated by comparing the dialogues recorded by the microphones located on the bridge to the values of the rudder angle entered by the helmsman at each instant. From a forensic point of view, the problem was to ascertain whether this error has or not been determinant for the impact. Our target was then to find out whether it was possible to avoid the impact had the captain's orders been executed correctly.

For a correct reconstruction of the facts, it was very important to evaluate the exact time at which the hull hit the rocks, as well as the exact contact point with the shallows, in the coordinate system of the ship's GPS. In fact, an accurate timereconstruction is crucial, due to the short time elapsed between the error and the impact. The Judge's consultants had previously performed an evaluation of the ship's position at the estimated impact time (21.45'.07''). This reconstruction, in our opinion, was not consistent with cartographic data: at 21.45'.07', the position of the breach on the ship's bulkhead was about $40 \mathrm{~m}$ behind the shallows. To investigate this point in more depth, we performed an accurate analysis of position, heading and time data of the ship. From these data, it was possible to calculate the instantaneous (linear and angular) velocity and acceleration. These quantities are plotted in Figs, 3, 4, 5 over time ( $\mathrm{t}=0$ corresponds to $21.44^{\prime} .00$ ', local time). Fig. 3 shows the behavior of linear velocity. Fig. 4 shows the actual rudder angle $\phi$ (blue line) along with the rudder angle 
ordered by the Master (red line): the discrepancy is evident in the interval 52-72s.

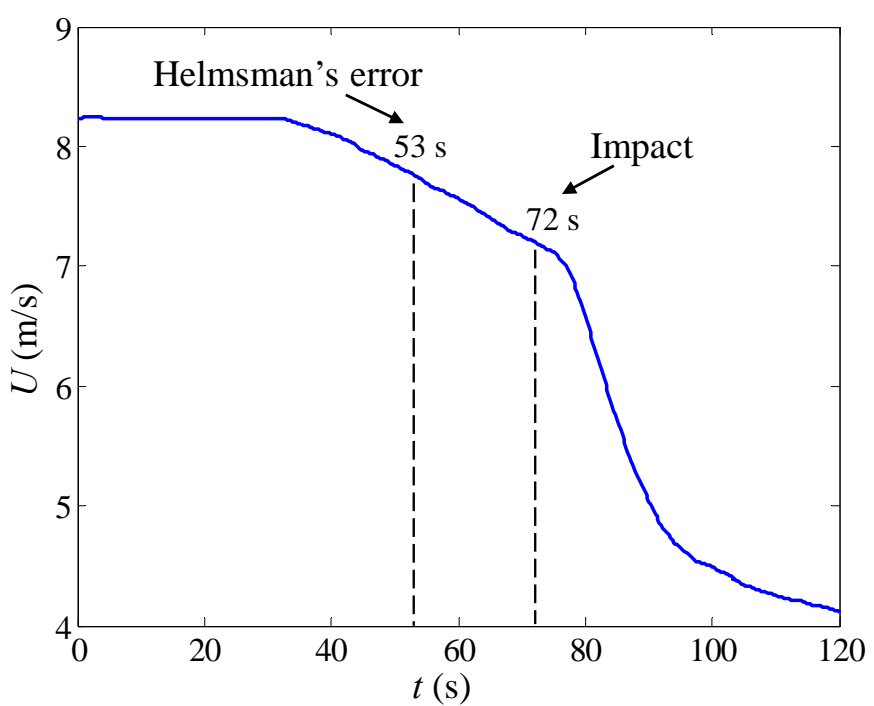

Fig. 3 Linear velocity of the ship. The star at $t=53 s$ corresponds to the helmsman's error; the one at $t=72 \mathrm{~s}$ to the impact

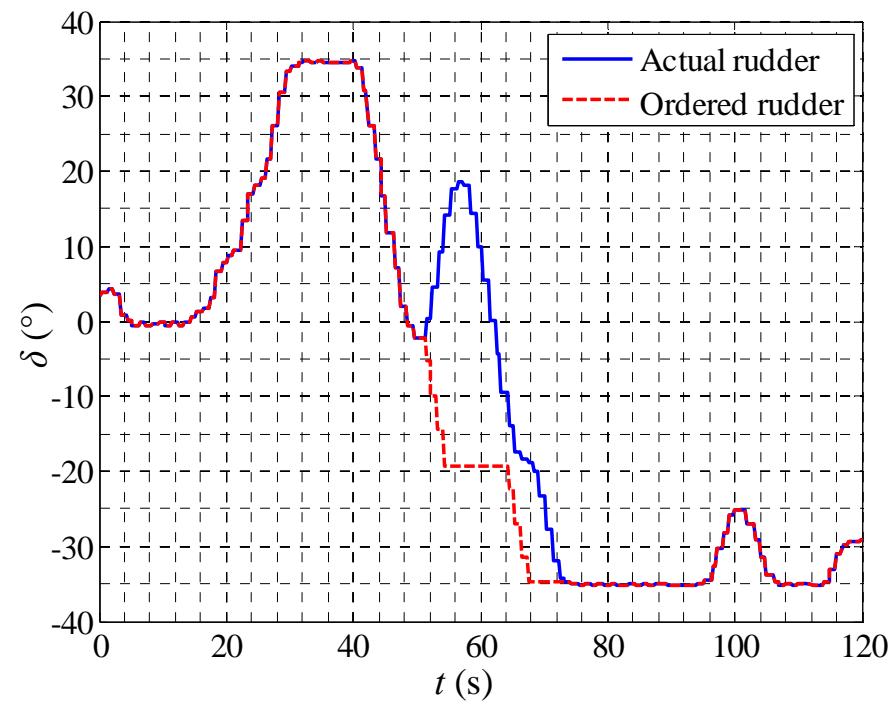

Fig.4 Actuated rudder angle: actual (blue) and as ordered (red)

From Figures 3 and 4 it is evident that when the rudder is away from its equilibrium position (i.e. $\phi=0$ ), the velocity decreases due to the fluid dynamic drag.

Figure 5 shows a plot of the acceleration: this is the most relevant data for the determination of the impact time. The figure shows that the impact can be positioned at 21.45'.12', (about 70 seconds into the plotted sequence), when a negative acceleration peak is observed, which lasts about 8s. This acceleration peak clearly indicates the time interval of the destructive contact with the rocks, in which about 500 MJ of kinetic energy of the ship were dissipated, in about 10s.
It is worth noting that the mean value of the ship velocity during the contact was about $6 \mathrm{~m} / \mathrm{s}$. At this speed, the ship sailed for about $48 \mathrm{~m}$ during the $8 \mathrm{~s}$ of the negative acceleration peak: this accurately corresponds to the length of the breach on the bulkhead of the ship.

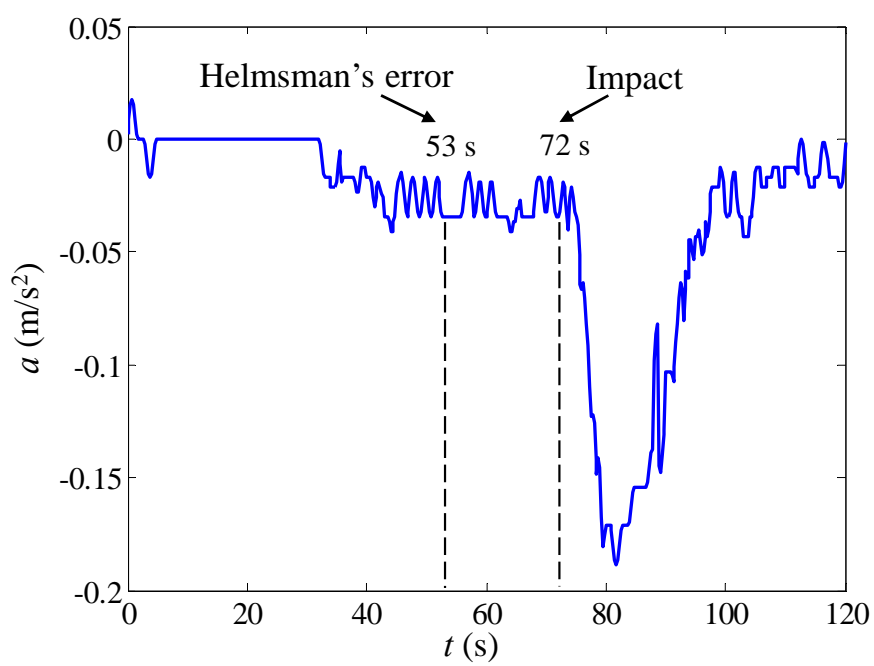

Fig. 5 linear acceleration of the ship. The star indicates the impact time.

From this analysis follows that the initial instant of the destructive contact has to be put at 21.45'.12', i.e. 5 s later than the impact time calculated by the Judge's consultants. This means that the ship was about $40 \mathrm{~m}$ ahead of the rocks when Schettino gave the order "hard to port" to the helmsman, who misunderstood it. The Judge's consultants, using qualitative arguments, stated that the helmsman's error had been inconsequential on the impact. This conclusion in our opinion should be reexamined using a quantitative approach and taking into account the aforementioned facts.

\section{EFFECTS OF THE HELMSMAN ERROR}

To investigate the effects of the helmsman's error on the impact with the rocks, a dedicated simulator was developed. The choice was for a short-term simulator, since the error occurred about 20s before the impact. This allowed to use a simplified model with only three degrees of freedom, neglecting roll and pitch angles and $z$ position. The effect of wind and current were also found to be negligible for a shortterm simulation. The hypothesis used to build the model and its mathematical formulation are described in [4]. The model's dynamic parameters were derived from known navigation data recorded during different manoeuvres, by means of a least square fit. This "black box" approach allows the model to learn the ship's response to stated inputs (i.e. rudder angle and propeller speeds).

The simulator was validated by comparing simulated trajectories with actual ones. To this end the data recorded in the VDR of the Costa Concordia during the last week of 
navigation before the accident were used. In the next phase, the final ship position and heading, computed by the simulator, were compared to data contained in the VDR, allowing us to evaluate the final error (the effect of the length of the simulated time interval on final accuracy was also evaluated). The simulator can also be used as a "maneuver predictor": further details are presented in [4, 5].

The model we just presented allowed us to study the maneuvers of the ship in proximity of the Giglio rocks. The impact time considered is 21.45'.12'”. Several simulations were performed, starting 30s ahead of the impact, just before the instant in which the captain planned a "Zig-Zag" maneuver as an extreme attempt to avoid the impact, ordering to put the rudders "hard to port". Data recorded in the VDR show that the helmsman misunderstood the order and instead set the rudders "hard to starboard" (Figure 4). To evaluate the effect of this error, the simulation was performed again, this time imposing the rudder position ordered by the captain, instead of the erroneous one. This rudder sequence was reconstructed using the recordings of the bridge audio stored in the VDR (Figure 4, red line).

Figure 6 shows the results obtained: the blue line represents the actual ship position at impact time (impact occurred) while the green line represents the simulated ship position, without the helmsman's error. The black ellipse represents the rock's position. As can be seen, in this case the left bulkhead of the ships is about $10 \mathrm{~m}$ from the rock. One could safely state that, according to the simulator, the ship would have passed close to the rock without impacting or, considering the worst case for the simulation error and model approximations, the impact could have occurred about $18 \mathrm{~m}$ behind.

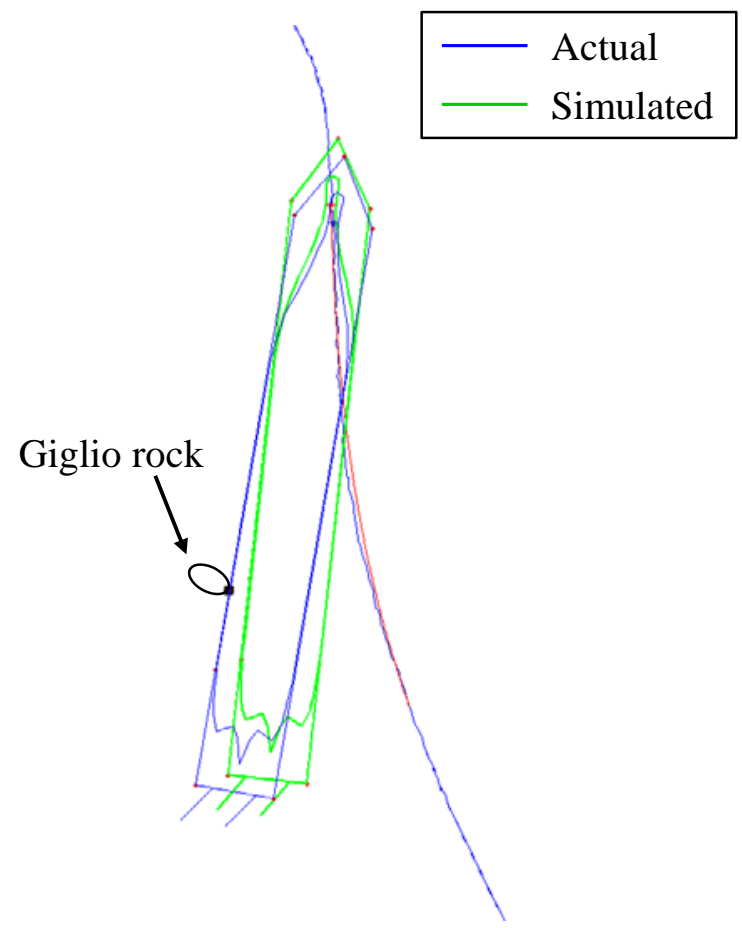

Fig. 6 Ship position at impact time without helmsman’s error.
Moreover, rotational speed of the ship would have been significantly lower (more than 50\%), causing a lower energy impact and consequently less serious damages to ship's hull. In the worst case, at least one engine room (and maybe both) would sustain no damage in the accident, allowing the ship to stay afloat and responsive to later commands. In this case, the final consequences would possibly have been different, especially in terms of lives lost.

\section{Automatic Emergency Procedure Malfunctionings}

Another field of investigation was the correct execution of the emergency procedure which automatically starts when a failure of the main power sources is detected by the automation system of the ship. Aboard the Costa Concordia the main source of energy was represented by six diesel generator of 14 MVA apparent power each, which stopped working just a few tens of seconds after the impact, due to the flooding of the machine rooms. At that point, an automatic procedure, named Emergency Shutdown, was scheduled to run, powered by the Emergency Diesel Generator (EDG) located at deck 11 of the ship, well above the sea level. Several witnesses reported that some emergency plants of the ship did not operate correctly or continuously after the impact. For instance, intermittent or lack of operation was allegedly experienced from emergency lights, emergency pump, passenger elevators and lifeboats winches. The cause or causes of these reported failures were not clear; in particular, it was not clear whether the EDG was operating or not. An analysis of the Martec SMS log files was then performed for the time starting at the impact (h 21.45.12) until the abandon of the ship (h 00.20.00). The results are summarized in Figure 7, where the events concerning not only the generator itself but also the EDG room, such as the ignition and shutoff of the fans, are reported.

As can be seen from a study of Figure 7, the EDG started at 21.46.16, stopped to run at 22.15.37, then started again and stopped after a few minutes of operation two more times, then definitively stopped at 22.36.46, well before the time at which the last lifeboats abandoned the ship. This fact could help explain the lack of power reported in some testimonies during the abandon of the ship: the EDG was not operating correctly.

Another point worth attention was the correct execution of the tests which have to be performed on EDG according to SOLAS (Safety Of Life At Sea) regulations and IMO (International Maritime Organization) resolutions. Since the data about the results of these test that were provided to the investigators apparently presented some inconsistencies, we decided to compare the information recorded in these files to what was stored in the hard disk of Martec SMS. Since Martec had provided only the data from $1 / 12 / 2012$ to $1 / 14 / 2012$, we tried to extract the relevant data from the previous log files by means of the technique illustrated in Section II. This allowed us to retrieve many pieces of information about the EDG starting from 12/25/2012: when the tests were performed, how many minutes they lasted, the events concerning the EDG room, such as the ignition and shutoff of the fans. As an 
example, in Figure 8 the events associated with the test performed on January 8, 2012, are shown. As can be seen, according to the data extracted, the EDG was started and then stopped twice, the first time after running for about 8 minutes, and the second after only 1'31'. Unfortunately, the data do not show why the EDG stopped the first time, nor whether the duration of the test was enough to allow the engine to attain its steady state temperature.

\begin{tabular}{|c|c|c|c|c|}
\hline Ora locale & Codice & Descrizione & Note & $\underline{\text { Stato }}$ \\
\hline 21.45 .18 & XA/1039 & Exhaust Fan & Ventola di estrazione & Stopped \\
\hline 21.45 .21 & VE/21138CS & Natural Supply Dumper & Serranda ingresso aria & Unknown \\
\hline 21.45 .33 & VE/21138CS & Natural Supply Dumper & Serranda ingresso aria & Closed \\
\hline 21.46 .02 & VE/21119CS & Exhaust Fan (Dumper) & Serranda ventola di estrazione & Unknown \\
\hline 21.46 .10 & EDDG & Emergency Diesel Generator & Generatore di Emergenza & Running \\
\hline 21.46 .40 & VE/21119CS & Exhaust Fan (Dumper) & Serranda ventola di estrazione & Open \\
\hline 21.46 .40 & VE/21141CS & Combustion Air Dumper (Radiator) & Serranda di ventilazione (radiatore) & Unknown \\
\hline 21.46 .49 & $\mathrm{VE} / 21119 \mathrm{CS}$ & Exhaust Fan (Dumper) & Serranda ventola di estrazione & Unknown \\
\hline 21.46 .57 & $\mathrm{XA} / 277$ & Fan & Ventola Generatore & Running \\
\hline 21.47 .02 & VE/21119CS & Exhaust Fan (Dumper) & Serranda ventola di estrazione & Closed \\
\hline 21.48 .23 & VE/21141CS & Combustion Air Dumper (Radiator) & Serranda di ventilazione (radiatore) & Open \\
\hline 21.55 .47 & $\mathrm{XA} / 277$ & Fan & Ventola Generatore & Stopped \\
\hline 22.15 .37 & EDDG & Emergency Diesel Generator & Generatore di Emergenza & Stopped \\
\hline 22.15 .38 & $\mathrm{VE} / 21141 \mathrm{CS}$ & Combustion Air Dumper (Radiator) & Serranda di ventilazione (radiatore) & Unknown \\
\hline 22.15 .48 & VE/21141CS & Combustion Air Dumper (Radiator) & Serranda di ventilazione (radiatore) & Closed \\
\hline 22.20 .16 & EDDG & Emergency Diesel Generator & Generatore di Emergenza & Running \\
\hline 22.20 .47 & $\mathrm{VE} / 21141 \mathrm{CS}$ & Combustion Air Dumper (Radiator) & Serranda di ventilazione (radiatore) & Unknown \\
\hline 22.22 .29 & $\mathrm{VE} / 21141 \mathrm{CS}$ & Combustion Air Dumper (Radiator) & Serranda di ventilazione (radiatore) & Open \\
\hline 22.28 .01 & EDDG & Emergency Diesel Generator & Generatore di Emergenza & Stopped \\
\hline 22.28 .03 & VE/21141CS & Combustion Air Dumper (Radiator) & Serranda di ventilazione (radiatore) & Unknown \\
\hline 22.28 .14 & VE/21141CS & Combustion Air Dumper (Radiator) & Serranda di ventilazione (radiatore) & Closed \\
\hline 22.30 .04 & EDDG & Emergency Diesel Generator & Generatore di Emergenza & Running \\
\hline 22.30 .36 & VE/21141CS & Combustion Air Dumper (Radiator) & Serranda di ventilazione (radiatore) & Unknown \\
\hline 22.32 .21 & $\mathrm{VE} / 21141 \mathrm{CS}$ & Combustion Air Dumper (Radiator) & Serranda di ventilazione (radiatore) & Open \\
\hline 22.36 .46 & EDDG & Emergency Diesel Generator & Generatore di Emergenza & Stopped \\
\hline 22.36 .47 & VE/21141CS & Combustion Air Dumper (Radiator) & Serranda di ventilazione (radiatore) & Unknown \\
\hline 22.36 .58 & $\mathrm{VE} / 21141 \mathrm{CS}$ & Combustion Air Dumper (Radiator) & Serranda di ventilazione (radiatore) & Closed \\
\hline
\end{tabular}

Fig. 7 EDG events after the impact until final blackout.

\begin{tabular}{|c|c|c|c|c|}
\hline Ora locale & Codice & Descrizione & Note & Stato \\
\hline $09: 40: 00$ & XA/1039 & Exhaust Fan & Ventola di estrazione & Stopped \\
\hline $09: 40: 03$ & VE/21119CS & Exhaust Fan (Dumper) & Serranda ventola di estrazione & Unknown \\
\hline 09:40:03 & $\mathrm{VE} / 21138 \mathrm{CS}$ & Natural Supply Dumper & Serranda ingresso aria & Unknown \\
\hline $09: 40: 12$ & EDDG & Emergency Diesel Generator & Generatore di Emergenza & Running \\
\hline $09: 40: 15$ & $\mathrm{VE} / 21119 \mathrm{CS}$ & Exhaust Fan (Dumper) & Serranda ventola di estrazione & Closed \\
\hline $09: 40: 32$ & $\mathrm{VE} / 21119 \mathrm{CS}$ & Exhaust Fan (Dumper) & Serranda ventola di estrazione & Unknown \\
\hline $09: 40: 42$ & $\mathrm{VE} / 21141 \mathrm{CS}$ & Combustion Air Dumper (Radiator) & Serranda di ventilazione (radiatore) & Unknown \\
\hline $09: 40: 56$ & XA/277 & Fan & Ventola Generatore & Running \\
\hline $09: 41: 44$ & XA/1039 & Exhaust Fan & Ventola di estrazione & Running \\
\hline $09: 42: 18$ & VE/21119CS & Exhaust Fan (Dumper) & Serranda ventola di estrazione & Open \\
\hline $09: 42: 25$ & VE/21141CS & Combustion Air Dumper (Radiator) & Serranda di ventilazione (radiatore) & Open \\
\hline 09:46:09 & XA/1039 & Exhaust Fan & Ventola di estrazione & Stopped \\
\hline $09: 46: 12$ & VE/21119CS & Exhaust Fan (Dumper) & Serranda ventola di estrazione & Unknown \\
\hline $09: 46: 30$ & VE/21119CS & Exhaust Fan (Dumper) & Serranda ventola di estrazione & Open \\
\hline $09: 47: 43$ & $\mathrm{VE} / 21138 \mathrm{CS}$ & Natural Supply Dumper & Serranda ingresso aria & Unknown \\
\hline $09: 48: 13$ & XA/1039 & Exhaust Fan & Ventola di estrazione & Running \\
\hline $09: 48: 46$ & EDDG & Emergency Diesel Generator & Generatore di Emergenza & Stopped \\
\hline $09: 48: 46$ & $\mathrm{XA} / 277$ & Fan & Ventola Generatore & Stopped \\
\hline $09: 48: 49$ & $\mathrm{VE} / 21141 \mathrm{CS}$ & Combustion Air Dumper (Radiator) & Serranda di ventilazione (radiatore) & Unknown \\
\hline $09: 49: 00$ & VE/21141CS & Combustion Air Dumper (Radiator) & Serranda di ventilazione (radiatore) & Closed \\
\hline $09: 49: 44$ & VE/21138CS & Natural Supply Dumper & Serranda ingresso aria & Operator Ack. \\
\hline $09: 50: 03$ & $\mathrm{VE} / 21138 \mathrm{CS}$ & Natural Supply Dumper & Serranda ingresso aria & Fault \\
\hline $09: 50: 13$ & $\mathrm{VE} / 21138 \mathrm{CS}$ & Natural Supply Dumper & Serranda ingresso aria & Alarm Ack. \\
\hline $09: 54: 48$ & EDDG & Emergency Diesel Generator & Generatore di Emergenza & Running \\
\hline $09: 54: 57$ & $\mathrm{VE} / 21141 \mathrm{CS}$ & Combustion Air Dumper (Radiator) & Serranda di ventilazione (radiatore) & Unknown \\
\hline $09: 55: 31$ & $\mathrm{XA} / 277$ & Fan & Ventola Generatore & Running \\
\hline $09: 56: 28$ & XA/277 & Fan & Ventola Generatore & Stopped \\
\hline $09: 56: 29$ & EDDG & Emergency Diesel Generator & Generatore di Emergenza & Stopped \\
\hline $09: 56: 36$ & $\mathrm{VE} / 21141 \mathrm{CS}$ & Combustion Air Dumper (Radiator) & Serranda di ventilazione (radiatore) & Closed \\
\hline
\end{tabular}

Fig. 8 EDG periodic test performed on Jan 8, 2012 


\section{CONCLUSIONS}

In this paper we have shown a typical case study, related to the reconstruction of the events in a disaster involving more than 4,000 people, in which most of the information was recorded in the memory devices (solid state memories and hard disks) of a complex information system. The results summarized in the paper demonstrate that this kind of information in some cases represents the only valuable source of data, not only for forensics purposes, but also for further scientific investigations. In the case described in the paper, the shipwreck of the Costa Concordia cruise ship, this was the only reliable way to attempt an accurate description of the dynamics of the accident and of the events immediately following the impact with Isola del Giglio's rocks. By using these data it was also possible to ascertain whether other responsibilities in addition to those of the captain, had to be investigated for a complete reconstruction of the casualty.

\section{ACKNOWLEDGMENT}

The authors are grateful to CODACONS (an Italian consumers' rights association), which assisted some of the shipwreck victims during the trials and made the data available.

\section{REFERENCES}

[1] National Marine Electronics Association (NMEA), "NMEA 0183 Standard” $\quad$ Retrieved 2013. http://www.nmea.org/content/nmea_standards/nmea_0183_v_410.asp

[2] Piccinelli,M., Gubian, P., "Modern Ships Voyage Data Recorders: a Forensics Perspective on the Costa Concordia Shipwreck", Proc. DFRWS Digital Forensics Research Workshop, Monterey CA (USA), Aug. 4-7 2013, also published in Digital Investigations, 10 (2013) S41-S49, Elsevier.

[3] Schneier, B., “Applied Cryptography”, John Wiley and Sons, 2nd Ed., 1996

[4] Neri, P., Piccinelli, M., Gubian, P., Neri, B., “A Ship Motion Short Term Time Domain Simulator and Its Application to Costa Concordia Emergency Manoeuvres just Before the January 2012 Accident”, Proc. ECMS European Conference on Modelling and Simulation 2014, Brescia, Italy, May 27-30 2014

[5] Neri, P., Neri, B., "A short term simulator for vessel manoeuvres prediction”, APPLEPIES 2015 Workshop, May 2015, Rome (to be published). 